\title{
The latent gram-negative bacterium Brucella induced different immune responses to general gram-negative bacterium Escherichia coli
}

\author{
International Journal of Infectious Diseases and Research
}

Research Article

Zheng Wang ${ }^{1,2^{*}}$, Guodong Xing ${ }^{1,2}$, Huawei Xie ${ }^{1,2}$, Zhou Shen ${ }^{1,2}$, Dongxia Zhang ${ }^{3}$, Xuebo Wang ${ }^{3}$, Qiao Xia, Qianqing Tang ${ }^{3}$, Junming $\mathrm{Li}^{3}$, Long Zhang ${ }^{4}$ and Qi Zhao ${ }^{3,4 \#}$

${ }^{1}$ Department of Pediatric General Surgery, Department of

Pediatrics, Chinese PLA General Hospital, Beijing, China.

${ }^{2}$ Department of Pediatric General Surgery, BaYi Children's Hospital, Seventh Medical Center of Chinese PLA General Hospital, Beijing, China. ${ }^{3}$ Yuhuangding Hospital, Shandong Province, Yantai, China,
264000 .

${ }^{4}$ Xiaoji Hospital of Haiyang, Shandong Province, Yantai, *Correspondence authors

Dr. Qi Zhao

20 Yuhuangding East Road

Zhifu District

Yantai, 264000

China

Submitted : 04 Jun 2021 ; Published : 28 Jun 2021 China, 264000.

\begin{abstract}
Brucellosis is one of the most common zoonoses in the world, and no effective methods for Brucella clearance completely until now. Therapy of brucellosis requires deeply understanding of mechanism of Brucella infection and immune responses. We collected human blood samples to analyze the difference of immune responses between latent gram-negative bacterium Brucella and general gram-negative bacterium Escherichia coli. To analyze cytokines release in plasma from patients, a multiplex MAP human cytokine/chemokine immunoassay was used. Human coagulation factor XI expression was detected using ELISA following instructions. In comparison with samples from patients infected with latent gram-negative bacterium Brucella, cytokines secreted by Th2 cells increased in patients infected with general gram-negative bacteria Escherichia coli, and also the chemokines, such as monocyte chemotactic protein 1(MCP-1), macrophage inflammatory protein (MIP-1 $\alpha, M I P-1 \beta)$. In the other side, in comparison with samples from patients infected with general gram-negative bacterium Escherichia coli, Interferon inducible protein 10 (IP-10) increased in patients infected with latent gram-negative bacterium Brucella, accompany with increased IFN- $\gamma$ secretion. Meanwhile, we also found only bacterium Brucella can increased factor XI secretion. Understanding immune response and coagulation function during bacterium Brucella infection will help us to find effective methods for Brucella clearance.
\end{abstract}

Keywords: Brucella; gram-negative bacterium; cytokine; Factor XI.

\section{Introduction}

Brucellosis, caused by species of the gram-negative, latent bacterium Brucella, continues to be a problem all over the world. The antibiotic can kill the extracellular Brucella, but failed when bacteria enter cells $[1,2]$. About vaccine, attenuated strains such as Brucella melitensis Rev1 and Brucella abortus S19 and RB51 are being used to control brucellosis in domestic animals [3]. However, no safe, effective vaccine is available for human use. Understanding natural, human immune responses to this species will help us to cure brucellosis.

In our study, we collected more than 50 human blood samples to analyze the difference of immune responses between latent gram-negative bacterium Brucella and general gram-negative bacterium Escherichia coli. We found that cytokines secreted by $\mathrm{Th} 2$ cells increased in patients infected with general gram-negative bacteria Escherichia coli, and also the chemokines, such as monocyte chemotactic protein 1 (MCP-1), macrophage inflam- matory protein (MIP-1 $\alpha$, MIP-1 $\beta$ ) increased in these patients. In the other side, Interferon inducible protein 10 (IP-10) increased in patients infected with latent gram-negative bacterium Brucel$l a$, accompany with increased IFN- $\gamma$ secretion. The coagulation is one part of innate immune responses, includes extrinsic pathway and intrinsic pathway. Both Escherichia coli and Brucella can active extrinsic pathway through tissue factor (TF). How about the intrinsic pathway? Here, we detected the levels of Factor XI in human blood, which is involved in intrinsic pathway of coagulation. We found only Brucella can increased factor XI secretion, may active intrinsic pathway and fibrin deposition. We believe these results will help us to have a deep understanding the mechanism of host immune response to bacterium Brucella.

\section{Results}

Patients were recruited for the study between September 2014 and September 2016. All 41 patients included in the study were judged I J Infectious Disea; $2021 \quad$ www.unisciencepub.com Volume 2| Issue 2 
to have an infection by the attending physician. The patients were grouped based on infection type. Thirty patients were female and 11 patients were male. The mean age was 43 years (range, 23-60 years). The healthy control group included 6 men and 10 women with a mean age of 44 years. Most patients did not have any apparent disorders.

To evaluate human immune responses, the blood is the sample we can get easily. Here, the Luminex technique was used to detected immune factors in human blood. More than 20 immune factors were assayed one time.

The same immune responses induced by Brucella and Escherichia coli

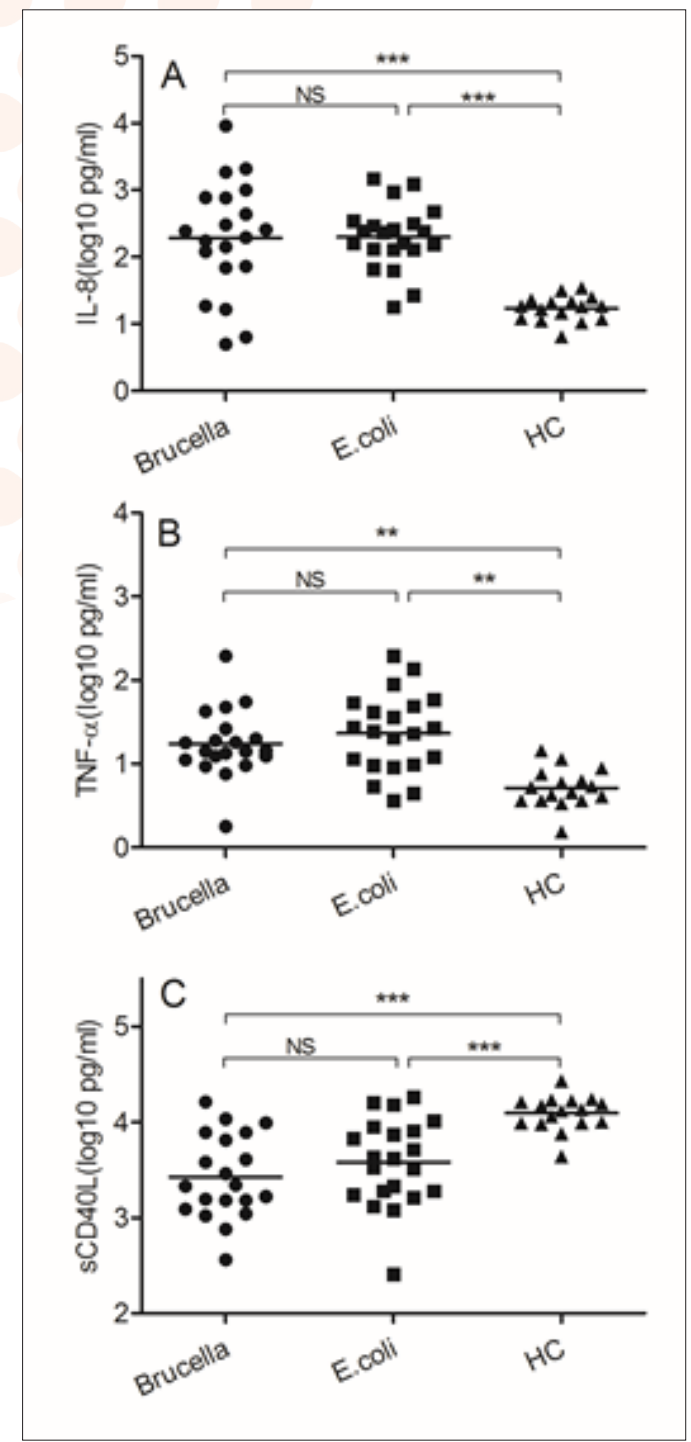

Figure 1: The same immune responses induced by Brucella and Escherichia coli

Blood samples were obtained on the day of admission to the hospital. (A)IL-8, (B) TNF- $\alpha$, (C) soluble CD40 ligand. We chose samples from healthy controls and patients infected with Brucella and Escherichia coli. Significant differences are indicated $(* * \mathrm{p}<0.005, * * * \mathrm{p}<0.001$, NS, no significant). All data are represented as mean $\pm \mathrm{SD}$. HC, Health Control.
As the gram-negative bacterium, both Brucella and Escherichia coli increased IL-8 secretion $(\mathrm{p}<0.0001)$, which can active and attractive neutrophils to infection sites. Since the neutrophil is important for gram-negative bacterium infection, TNF- $\alpha$ also increased in two kind of bacterium $(\mathrm{p}<0.005)$. TNF- $\alpha$ can increased phagocytic ability of neutrophil. The soluble CD40 ligand, also named TNF associated activation protein, expressed on surface of CD4 T cells, increased in both bacteria $(\mathrm{p}<0.0001)$. Like the general gram-negative bacterium Escherichia coli infection, we believe neutrophil and CD4 $\mathrm{T}$ cell also play important roles in Brucella infection. Unlike other extracellular bacterial pathogens, the host resistance to Brucella depends mainly on acquired cell-mediated immunity. Next, we assessed the different immune responses induced by these two bacteria.

Different immune responses induced by Brucella and Escherichia coli
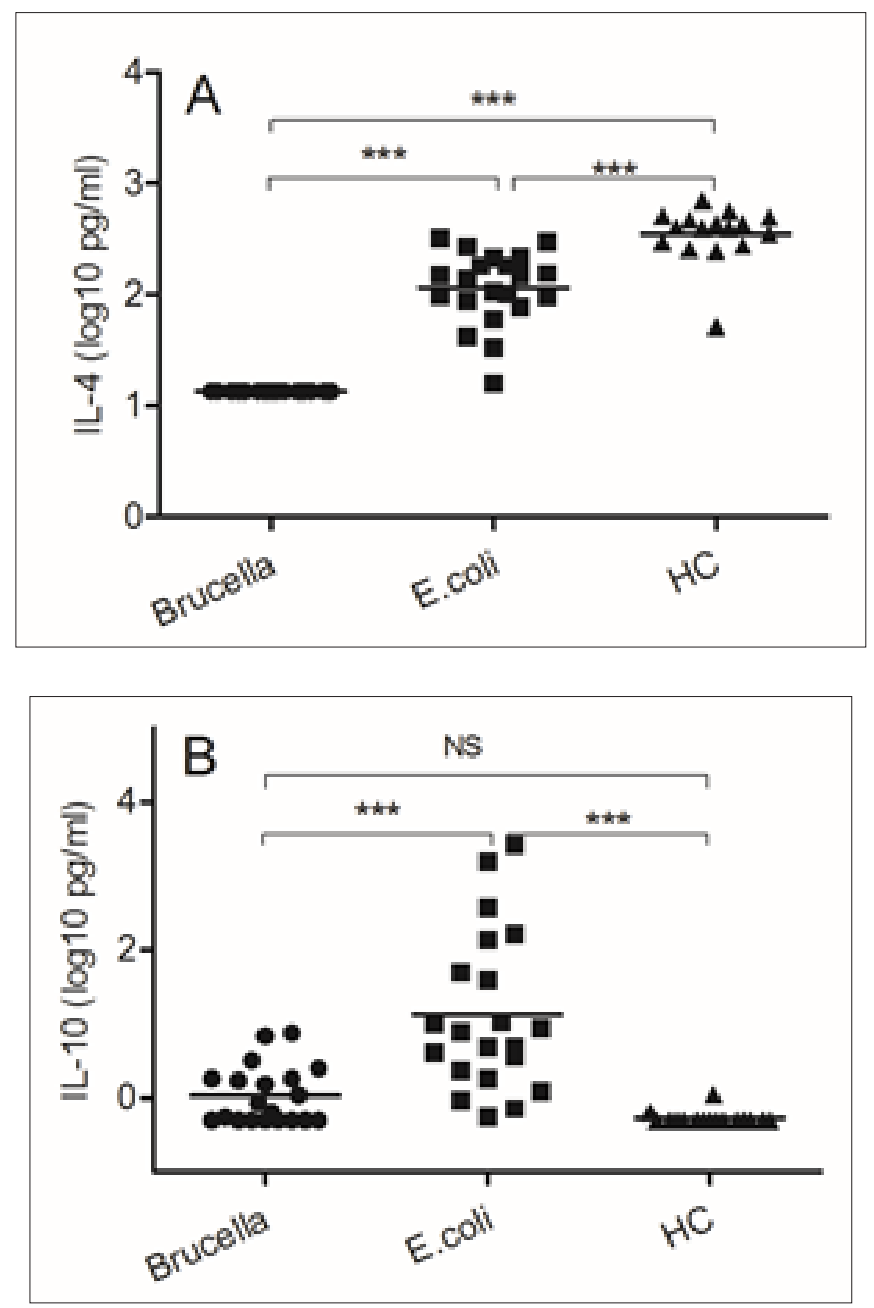

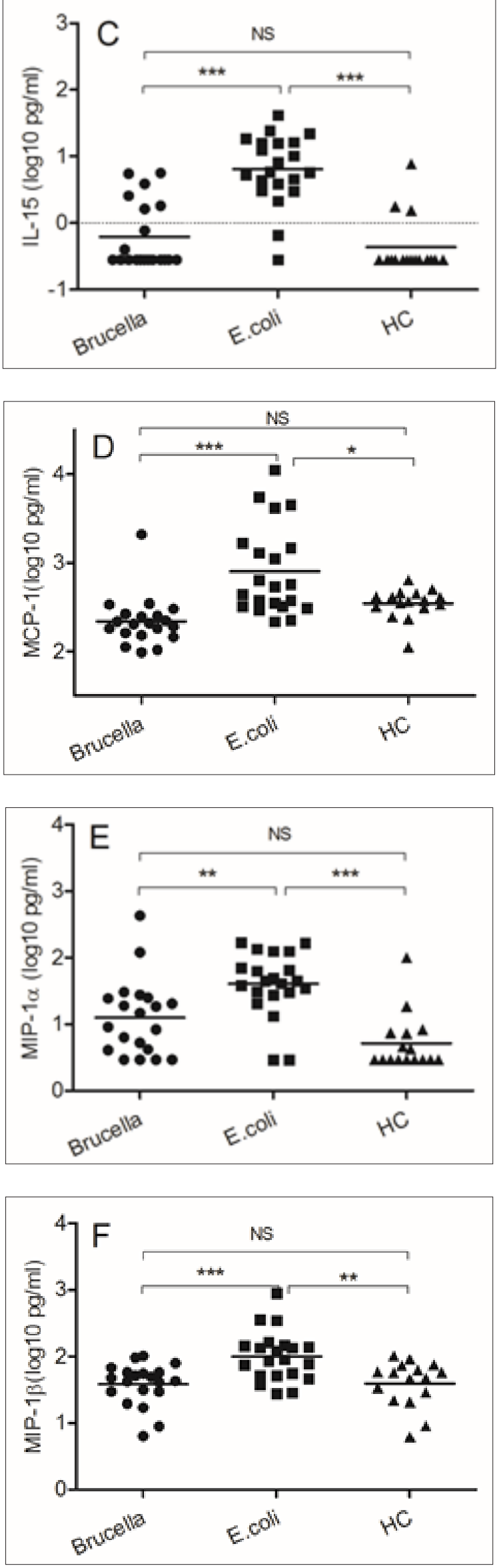
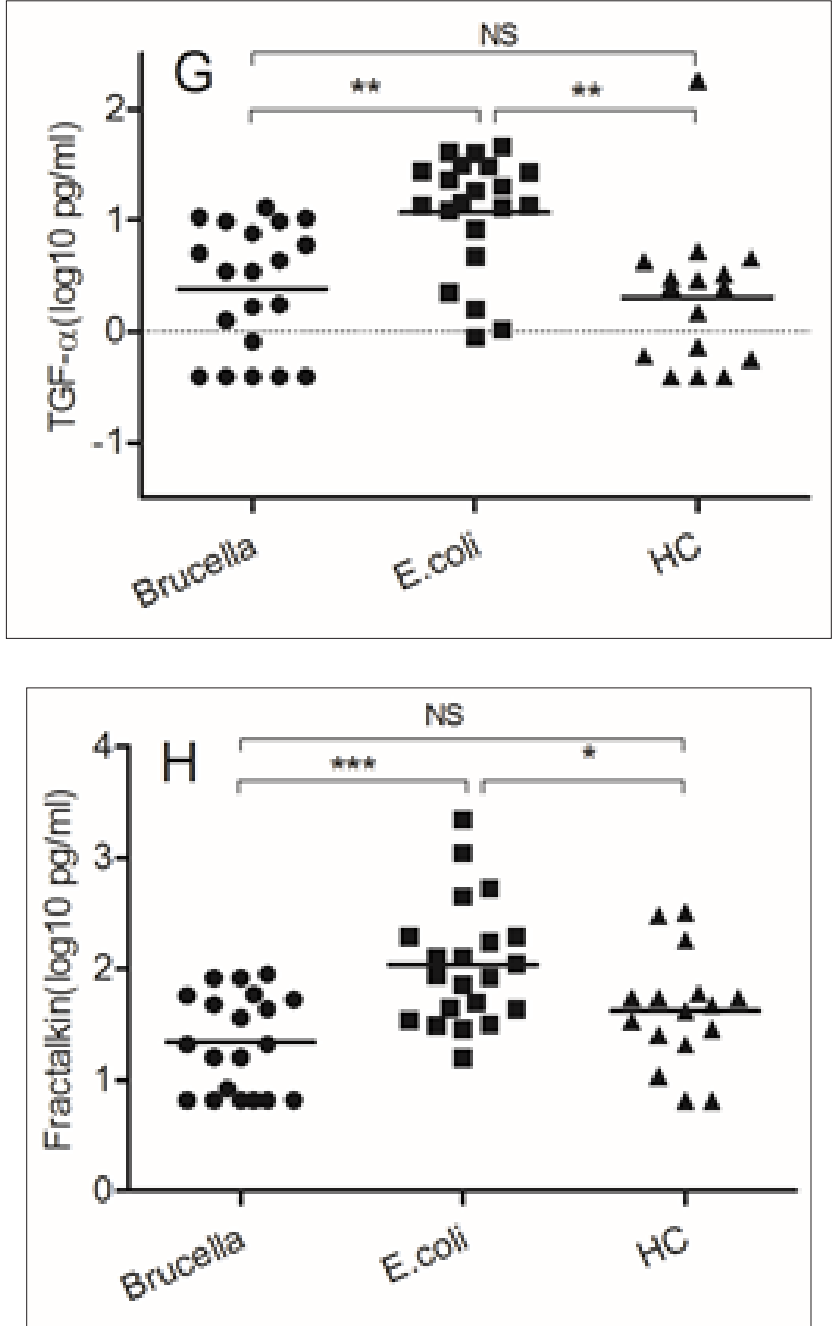

Figure 2: Different immune responses induced by Brucella and Escherichia coli

Blood samples were obtained on the day of admission to the hospital. (A)IL-4, (B) IL-10, (C) IL-15, (D)MCP-1, (E)MIP$1 \alpha,(\mathrm{F}) \mathrm{MIP}-1 \beta,(\mathrm{G}) \mathrm{TGF}-\alpha,(\mathrm{H})$ Fractakine. We chose samples from healthy controls and patients infected with Brucella and Escherichia coli. Significant differences are indicated $(* * \mathrm{p}<0.005, * * * \mathrm{p}<0.001, \mathrm{NS}$, no significant). All data are represented as mean $\pm \mathrm{SD}$. HC, Health Control.

The gram-negative bacterium Escherichia coli is an extracellular pathogen. The host resistance to Escherichia coli depends mainly on antibody-mediated immunity. In comparison with samples from patients infected with Brucella, we found increased IL-4 $(\mathrm{p}<0.0001)$ and IL-10 $(\mathrm{p}<0.0001)$ expression in Escherichia coli infected samples, and the function of this kind of Th2 type cytokines is stimulating B cell to secret antibody, such as IgG and IgE. In our study, we also detected higher levels of IL-15 $(\mathrm{p}<0.0001)$ in blood of patients infected with Escherichia coli even though we did not uncertain what that means. In comparison with samples from patients infected with Brucella, we detected higher levels of monocyte chemotactic protein $1(\mathrm{MCP}-1)(\mathrm{p}<0.0001)$ and macrophage inflammatory protein (MIP-1 $\alpha$, MIP-1 $\beta$ ) in samples of patient infected with Escherichia coli. We believe the monocyte and macrophage 
are essential for most extracellular pathogens, which supply the first defense to this kind of bacteria. Meanwhile, we detected some other factors increased in samples of patient infected with Escherichia coli in comparison with samples from patients infected with Brucella, such as transforming growth factor $\alpha($ TGF- $\alpha)(p<0.005)$ and Fractakine $(p<0.0001)$.

Latent garm-negative bacterium Brucella induced higher levels of IFN- $\gamma$ secretion

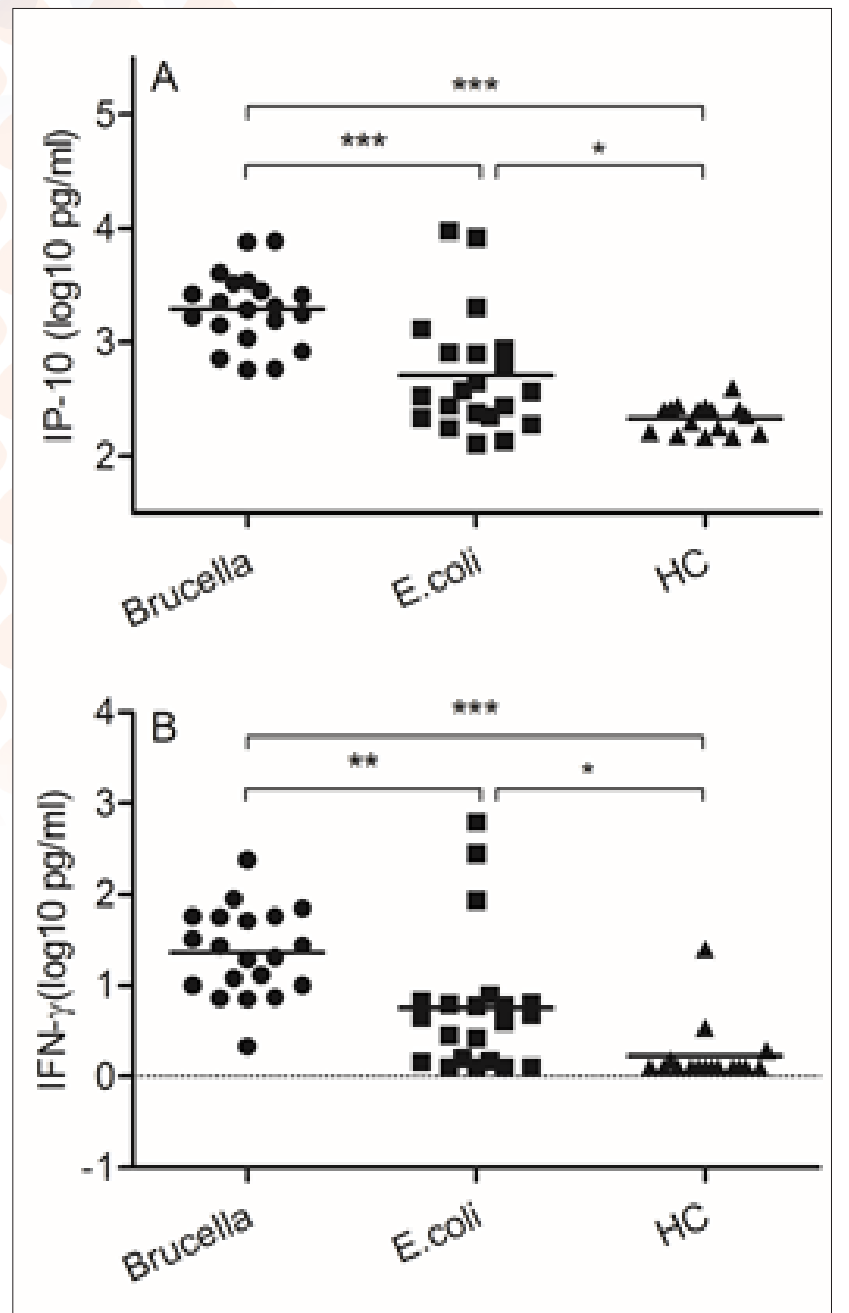

Figure 3: Brucella induced higher levels of IP-10 and IFN- $\gamma$ secretion

Blood samples were obtained on the day of admission to the hospital. (A)IP-10, (B) IFN- $\gamma$. We chose samples from healthy controls and patients infected with Brucella and Escherichia coli. Significant differences are indicated $(* * \mathrm{p}<0.005$, $* * * p<0.001$, NS, no significant). All data are represented as mean \pm SD. HC, Health Control.

Brucella abortus is a facultative intracellular bacterial pathogen responsible for brucellosis. The development of a Th1 subset of CD4 and CD8 lymphocytes secreting gamma interferon (IFN- $\gamma$ ), a crucial cytokine that can up regulate the anti-Brucella activity of macrophages (14). In this study, we evaluated levels of interferon inducible protein 10 (IP-10) and IFN- $\gamma$. In comparison with samples from patients infected with Escherichia coli, we found increased IP-10 $(\mathrm{p}<0.0001)$ and IFN- $\gamma$ $(p<0.005)$ expression in Brucella infected samples.

Latent gram-negative bacterium Brucella induced higher levels of Factor XI secretion, may active intrinsic pathway of coagulation

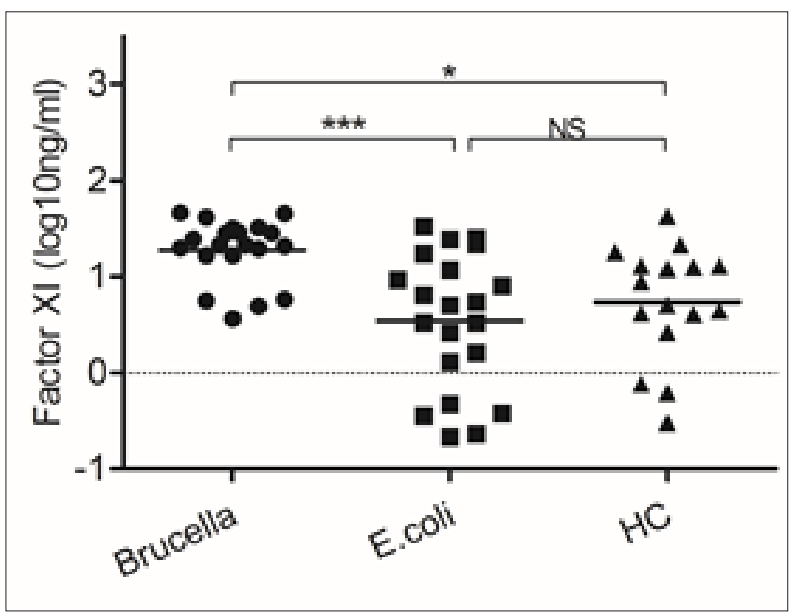

Figure 4: Brucella induced higher levels of Factor XI secretion

Blood samples were obtained on the day of admission to the hospital to detect levels of Factor XI. We chose samples from healthy controls and patients infected with Brucella and Escherichia coli. Significant differences are indicated $(* * * p<0.001$, $\mathrm{NS}$, no significant). All data are represented as mean \pm SD. HC, Health Control.

Coagulation cascade includes two pathways, intrinsic and extrinsic pathway. The initiating elements of the intrinsic pathway are not thought to play a major role during hemostasis in response to vascular trauma, but infectious agents are known to trigger both the intrinsic and extrinsic pathway $[4,5]$. Here we detected two factors involved in the intrinsic and extrinsic pathways respectively. The same levels of tissue factor were detected in samples of patients infected with Escherichia coli and Brucella (data not shown). Nevertheless, Factor XI, one important factor of intrinsic pathway, increased in samples of patients infected with Brucella $(\mathrm{p}<0.0001)$.

\section{Discussion}

Brucella abortus is a facultative intracellular pathogen and one of the etiological agents of brucellosis that can infect humans and domestic animals. Because this kind of bacterium can escape form host immunity and survival in cells, antibiotic therapy is long and costly, and does not work [6]. Meanwhile, there is no safe and effective vaccine for human use [6]. To understand the mechanism of host immune response to Brucella will help us to find a new method for brucellosis treatment. Here, we collected more than 50 human blood samples to test. These results show that both the immune response and coagulation function displayed significant difference between latent gram-negative bacterium Brucella and general gram-negative bacterium Escherichia coli.

First, we assessed the same immune responses induced by Brucella and Escherichia coli. As the gram-negative bacteri- 
um, they both increased IL- 8 and TNF- $\alpha$ secretion, which can active and attractive neutrophils to infection sites. We know that the neutrophil is important for gram-negative bacterium infection. The soluble CD40 ligand, also named TNF associated activation protein, expressed on surface of CD4 T cells, increased in both bacteria [1]. The second, we assessed the different immune responses induced by Brucella and Escherichia coli. Like other intracellular bacterial pathogens, the host resistance to Brucella depends mainly on acquired cell-mediated immunity. Whereas, the host resistance to Escherichia coli depends mainly on antibody-mediated immunity. In comparison with samples from patients infected with Escherichia coli, we found decreased IL-4 and IL-10 expression in Brucella infected samples, and the function of this kind of Th2 type cytokines is stimulating B cell to secret antibody, such as $\operatorname{IgG}$ and $\operatorname{IgE}[7,8]$. Meanwhile, In comparison with samples from patients infected with Escherichia coli, we detected lower levels of monocyte chemotactic protein 1 (MCP-1) and macrophage inflammatory protein in samples of patient infected with Brucella. We believe the monocyte and macrophage are essential for most extracellular pathogens, but not intracellular pathogens, which supply the first defense to this kind of bacteria [9]. Brucella abortus is a facultative intracellular bacterial pathogen responsible for brucellosis [6]. The development of a Th1 subset of CD4 and CD8 lymphocytes secreting gamma interferon (IFN- $\gamma$ ), a crucial cytokine that can up regulate the anti-Brucella activity of macrophages[10, 11]. In this study, we detected higher levels of interferon inducible protein 10 (IP-10) and IFN- $\gamma$ during Brucella infection.

Fibrin performs critical protective roles during infections, such as restricting bacterial dissemination, recruiting neutrophils to clear microorganisms, and facilitating $\mathrm{T}$ cell function $[4,12]$. However, we observed that a very high level of fibrin played a negative role during infection, in addition to that of thrombosis [13]. In our previous study, we emphasize that only the appropriate level of fibrin confers protection [14]. The classical "extrinsic" coagulation cascade is driven by the exposure of plasma to tissue factor, which facilitates the activation of factor VII (FVII), FIX, FX, and prothrombin, in turn leading to the generation of thrombin, the activation of platelets, and the feedback activation of factors XI, V, and VIII, further accelerating thrombin generation and, ultimately, prompting the deposition of insoluble fibrin, a structural component of the blood clot [15]. So we hypothesized that "good" fibrin deposit when the extrinsic pathway was activated, whereas, the "bad" fibrin deposit when intrinsic pathway was activated. In this study, we detected two factors involved in the intrinsic and extrinsic pathways respectively. The same levels of tissue factor were detected in samples of patients infected with Escherichia coli and Brucella. Nevertheless, Factor XI $[16,17]$, one important factor of intrinsic pathway, increased in samples of patients infected with Brucella. This higher level of factor XI secretion may active intrinsic pathway and "bad" fibrin deposition. We still do not know the protective role of fibrin during Brucella infection. We hypothesized that the "bad" fibrin impacts the bacterium invasion, which increased the ability to survival in cells. In order to find the relationship between Brucella infec- tion and coagulation, our further plan for this study will focus on mechanism research in mouse models.

Understanding immune responses and coagulation function during Brucella infection will help us to find a new way to cure this kind of latent bacterium infection.

\section{Materials and Methods [18]}

The study protocol was approved by the ethics committee of General Hospital of PLA and Yuhuangding Hospital, and the patients or relatives provided informed consent.

\section{Patients and study design}

This study was investigated in 41 infected patients and 16 healthy volunteers. Cultured samples from general gram-negative bacterium Escherichia coli infected patients required more than 5 days, and samples with bacterial growth over ' +++ ' were chosen. Patients whose cultured samples grew diphtheroid rod or two or more bacterial species were excluded from the study. To identify the Brucella infection, Serum agglutination test was used in BLS-2, and the titer over 1:100 samples were chosen. Other exclusion criteria included: age less than 23 years or more than 60 years, heparin administration, use of drugs affecting prostanoid synthesis, hematological malignancies and use of cytostatic drugs. Healthy volunteers who had no evidence of infection and had not taken anti-platelet medication in the previous 20 days were recruited for the study.

\section{Blood collection and analysis}

Blood samples were obtained on the day of admission to the hospital. Two milliliters of blood were drawn in EDTA anticoagulant tubes (Liuyang ME Company, Hubei, China) by venipuncture. The plasma fraction was separated and stored at $-80^{\circ} \mathrm{C}$.

Bacterial identification and cullure of patient samples To identify the bacterial species in patient samples, the VITEK 2 system (VITEK 2 Compact 60, Marcy-l'Etoile, France) was used according to the operating instructions. To determine the abundance of general gram-negative bacterium Escherichia coli, samples were blotted onto the medium. Samples were collected if they registered at least ' +++ ' bacterial growth in 5 days, and samples were discarded if they registered less than ' +++ ' bacterial growth, grew diphtheroid rods or contained two or more bacterial species. To identify the Brucella infection, Serum agglutination test was used in BLS-2, and the titer over 1:100 samples were chosen, other inclusion criteria included: the temperature is higher than $37.8^{\circ} \mathrm{C}$; the number of white cell exceed $10 \times 109 / \mathrm{L}$ and no bacteria can be cultured in blood.

\section{Multiplex analysis (Luminex)}

To analyze cytokines release in plasma from patients, a multiplex MAP human cytokine/chemokine immunoassay (Millipore, Billerica, Massachusetts, USA) was used following the manufacturer's protocol (Luminex FLEXMAP 3DTM system, xPOMENT software, Millipore). Twenty-eight growth factors, inflammatory cytokines and chemokines were quantitatively analyzed. 
Indirect ELISA

Human coagulation factor XI expression (Abcam 108834, Hong Kong, China) in plasma was detected using ELISA following instructions. The absorbance was read on a microplate reader at a wavelength of $450 \mathrm{~nm}$.

\section{Statistical analysis}

Statistical analyses were performed using the program Prism 5.0 (GraphPad Software, Inc., La Jolla, California, USA). Values are expressed as mean \pm SD. Data were analyzed by unpaired Student's t-test (normal distribution) or one-way ANOVA. $\mathrm{P}<0.05$ was considered to be statistically significant.

\section{Acknowled gments}

The present work was supported by funding of Yantai Technology plan(2015WS043), Medical Science and Technology Development plan of Shandong(2019WS0038).

\section{Author Contributions}

All authors discussed the results and implications of the manuscript. Z.W and Q.Z. conceived the study, supervised the project, analyzed data, and wrote the paper. X.G., X.W., S.Z., Z.D., W.X., X.Q., T.Q., L.J. and Z.L. performed experiments and analyzed data. Z.W. advised on statistical evaluations.

\section{Conflict of interest}

There are no conflicts of interest.

\section{Ethics Statements}

We collected blood from 41 patients enrolled in PLA General Hospital and Yuhuangding Hospital from March 2018 to April 2019 after obtaining written informed consent from patients or their surrogates.

\section{Reference}

1. Diacovich L, Gorvel JP (2010). Bacterial manipulation of innate immunity to promote infection. Nature Reviews Microbiology, 8(2):117-128.

2. Musa MT, Shigidi MTA, Saeed OM (1999). Brucellosis in humans associated with animals. Saudi Medical Journal, 20(4):311-315.

3. Luo D, Bing N, Li P, Shi W, Zhang SL, Han Y, Mao LW, He YD, Wu YZ, Wang XL (2006). Protective immunity elicited by a divalent DNA vaccine encoding both the L7/ L12 and Omp16 genes of Brucella abortus in BALB/c mice. Infection and Immunity, 74(5):2734-2741.

4. Oehmcke S, Herwald H (2010). Contact system activation in severe infectious diseases. Journal of Molecular Medicine-Jmm, 88(2):121-126.

5. Tapper H, Herwald H (2000). Modulation of hemostatic mechanisms in bacterial infectious diseases. Blood, 96(7):2329-2337.

6. Vitry MA, De Trez C, Goriely S, Dumoutier L, Akira S, Ryffel B, Carlier Y, Letesson JJ, Muraille E (2012). Crucial Role of Gamma Interferon-Producing CD4(+) Th1 Cells but Dispensable Function of CD8(+) T Cell, B Cell, Th2, and Th17 Responses in the Control of Brucella melitensis Infection in Mice. Infection and Immunity,
$80(12): 4271-4280$.

7. Akira S (2009). Pathogen recognition by innate immunity and its signaling (vol 85, pg 143, 2009). Proceedings of the Japan Academy Series a-Mathematical Sciences, 85(6):216-216.

8. Akira S, Uematsu S, Takeuchi O (2006). Pathogen recognition and innate immunity. Cell, 124(4):783-801.

9. Akira S (2013). Pathogen recognition receptors and innate immunity. Xenotransplantation, 20(5):351-351.

10. Kanangat S, Meduri GU, Tolley EA, Patterson DR, Meduri CU, Pak C, Griffin JP, Bronze MS, Schaberg DR (1999). Effects of cytokines and endotoxin on the intracellular growth of bacteria. Infection and Immunity, 67(6):28342840.

11. Dorneles EMS, Teixeira-Carvalho A, Araujo MSS, Sriranganathan N, Lage AP (2015). Immune response triggered by Brucella abortus following infection or vaccination. Vaccine, 33(31):3659-3666.

12. Luo DY, Lin JS, Parent MA, Mullarky-Kanevsky I, Szaba FM, Kummer LW, Duso DK, Tighe M, Hill J, Gruber A et al (2013). Fibrin Facilitates Both Innate and T Cell-Mediated Defense against Yersinia pestis. Journal of Immunology, 190(8):4149-4161.

13. Chapin JC, Hajjar KA (2015). Fibrinolysis and the control of blood coagulation. Blood Reviews, 29(1):17-24.

14. Zhao Q, Liu X, Zhang DX, Zhang LY, Luo DY (2015). Hexa-acylated LPS-lipid A deploys the appropriate level of fibrin to confer protection through MyD88. International Journal of Infectious Diseases, 33:142-148.

15. Mullarky IK, Szaba FM, Winchel CG, Parent MA, Kummer LW, Mackman N, Johnson LL, Smiley ST (2006). In situ assays demonstrate that interferon-gamma suppresses infection-stimulated hepatic fibrin deposition by promoting fibrinolysis. Journal of Thrombosis and Haemostasis, 4(7):1580-1587.

16. Bane CE, Boyd KL, Tucker EI, Smiley ST, Gruber A, Gailani D (2013). Factor XI Deficiency Reduces The Inflammatory Response To Polymicrobial Sepsis In Mice. Blood, 122(21).

17. Luo DY, Szaba FM, Kummer LW, Johnson LL, Tucker EI, Gruber A, Gailani D, Smiley ST (2012). Factor XI-Deficient Mice Display Reduced Inflammation, Coagulopathy, and Bacterial Growth during Listeriosis. Infection and Immunity, 80(1):91-99.

18. Wang Z, Zhao Q, Zhang DX, Sun CM, Bao CX, Yi ML, Xing L, Luo DY (2016). Essential roles for platelets during neutrophil-dependent or lymphocyte-mediated defense against bacterial pathogens. Blood Coagulation \& Fibrinolysis, 27(6):667-672.

Copyright: (C2021 Zheng Wang. This is an open-access article distributed under the terms of the Creative Commons Attribution License, which permits unrestricted use, distribution, and reproduction in anymedium, provided the original author and source are credited. 